\title{
Sol-gel processed Superhydrophobic Plastic Surfaces Modified with Perfluorooctyltriethoxysilane (POTS)
}

\author{
Natalia Picolo ${ }^{a}$, Viviane Tavares de Moraes ${ }^{b}{ }^{\oplus}$, Guilherme Wolf Lebrão $o^{b}$, \\ Susana Marraccini Giampietri Lebrã ${ }^{b *}$ (1) \\ ${ }^{a}$ Departamento de Engenharia Química, Centro Universitário do Instituto Mauá de Tecnologia, São \\ Caetano do Sul, SP, Brasil \\ ${ }^{b}$ Departamento de Engenharia Mecânica, Centro Universitário do Instituto Mauá de Tecnologia, São \\ Caetano do Sul, SP, Brasil
}

Received: August 27, 2019; Revised: December 16, 2019; Accepted: February 9, 2020

\begin{abstract}
Researches about nanomaterials related to properties such as superhydrophobicity, self-cleaning, corrosion and scratching resistance can be directly related to the materials' wettability. This characteristic is quantified by the contact angle made between the surface and a water droplet, in which angles above 90 degrees are considered to be hydrophobic and those above 150 degrees, superhydrophobic. The focus of this work was the development of a superhydrophobic self-cleaning surface, using silica nanoparticles with a particle diameter around $400 \mathrm{~nm}$ to $800 \mathrm{~nm}$ (produced via sol-gel, referring to the classic Stöber method) functionalized with perfluorooctyltriethoxysilane (POTS) on a polymeric substrate polymethyl methacrylate (PMMA). Four different surface treatment conditions were analyzed: untreated, treated with non-functionalized silica nanoparticles, treated only with POTS and treated with silica nanoparticles functionalized with POTS. For this last condition, a static water contact angle of $(150.0 \pm 0.44)$ degrees and a dynamic water contact angle of $(7.5 \pm 0.38)$ degrees were obtained, which is a typical value of a superhydrophobic surface. However, the surface treated with POTS alone had a contact angle of 115 degrees. The high contact angle value was due to the low surface energy obtained, which was of $(1.45 \pm 0.02) \mathrm{dyn} / \mathrm{cm}$. The modified superhydrophobic surface revealed a superior self-cleaning performance by freely rolling spherical water drops on the non-wettable solid surface. The stability of the modified surface has been proven by water jet impact.
\end{abstract}

Keywords: superhydrophobic, wettability, surface energy, silica nanoparticles, contact angle.

\section{Introduction}

The development of superhydrophobic nanomaterials is a tendency for applications in coatings in order to increase the toughness and corrosion, mechanical and scratching resistance. These materials can have this performance increase by simply decreasing their surface wettability ${ }^{1,2,3}$.

For a material to be classified as hydrophilic, it should present $\theta_{E} \leq 90^{\circ}$; for hydrophobic materials, $150^{\circ} \leq \theta_{E} \leq 90^{\circ}$; and for the superhydrophobic materials, $\theta_{E}>150^{\circ 4,5}$.

The use of hydrophobic nanomaterials has increased once the decrease in wettability helps to improve other properties. An example is the use of superhydrophobic nanomaterials on airplane surfaces and air conditioner and refrigerator tubes that cannot freeze when in touch with water ${ }^{6,7}$. The steel coating for the shipbuilding industry and for chirurgical instruments is also an application that stands out to prevent marine algae films and fixing bacteria on instruments in contact with body fluids ${ }^{8}$. Hydrophobic fabrics have also self-cleaning properties, which can be applied to upholstery, usual clothes, mattresses, and for fabric used for water sports clothes, which can favor the cleaning process, with low humidity, sweat absorption and reduced friction ${ }^{9,10}$. The applications are diverse, particularly

*e-mail: susana.lebrao@maua.br highlighting their use in automobile and aircraft paints and varnishes, pipe coating, hydrophobic fabrics and ophthalmic lenses ${ }^{11,12}$, oil spill clean-up from water ${ }^{13,14}$.

For a superhydrophobic silica nanoparticles synthesis, there are the pyrogenic and the precipitation methods more widespread than others, but their drawbacks are the lack of dispersion and nanoparticle size control.

The pyrogenic method produces a fine, white and amorphous silica particle by a high temperature steam process, in which tetrachlorosilane suffers oxidation by an oxygen and hydrogen flame. This is the most widely used process in the industry nowadays ${ }^{15,16}$.

The precipitation method is made by an aqueous process, starting from the silicates treatment with acids to obtain hydrated silica particles for precipitation. Yet this method generates more silanol groups on the surface of the particles $(\mathrm{Si}-\mathrm{OH})$ than the pyrogenic method which facilitates their agglomeration and, therefore, hinders the nanoparticles formation ${ }^{17}$.

Other methods, not yet widespread in the industry, are the microemulsion and sol-gel methods for silica nanoparticle fabrication.

These two methods can be alternatives for dispersion and nanoparticle size control. However, the microemulsion or emulsion method involves the addition of surfactant, which requires several washing steps before the use of these nanomaterials. 
Studies have shown that in the silica-nanoparticle fabrication process via emulsification, a mixture of surfactant (Berol 26, 160 and 267) and water was used, under constant stirring, with the addition of TEOS and hydrocarbons to promote the sample emulsion in alkaline conditions and with the addition of ammonium hydroxide using an ultrasound apparatus ${ }^{18}$.

In this microemulsion process, the surfactant molecules dissolved in organic solvents form spherical micelles. In the presence of water, the polar groups reorganize into microcavities containing water, which are called reverse micelles. The nanoparticles can be produced inside these micro-cavities by controlling the silicone alkoxides addition. The major disadvantage of this method is the high cost and the difficulty in removing the surfactants at the end of the process ${ }^{14,19}$.

The microemulsion process of silica-nanoparticle fabrication generates particles with higher agglomeration chances, especially if produced with high water content (above 20 wt.\%). The system with low water content (below 15 wt.\%) produces a more dispersed silica nanoparticle, but their size distribution cannot be fully controlled; the measured particle size stays between $10 \mu \mathrm{m}$ and $40 \mu \mathrm{m}$, but a uniform dispersion cannot be achieved ${ }^{17}$.

The process of obtaining silica nanoparticles via solgel method is simpler, since it considers hydrolysis of the suspended silica particles in the aqueous medium, in the presence of alcohol and hydroxide, but it can generate particles sized between $50 \mathrm{~nm}$ and $700 \mathrm{~nm}^{17}$.

In order to control the size and morphology of the particle generated in the sol-gel process, hydrolysis control can be adopted by diluting tetraorthosilicate (TEOS) in ethanol in alkaline conditions. Studies demonstrate the formation of particles in the range of $10 \mathrm{~nm}$ to $2 \mu \mathrm{m}^{14,15}$.

This sol-gel method is a two-stage formation process, in which the first is the hydrolysis of a metal alkoxide (known as nucleation) and the second a polycondensation (growth) reaction.

The major interest of this method is its first step, in which silica forms a network of oxides in an organic matrix. During this step, the hydrolysis of TEOS molecules $\left(\mathrm{Si}\left(\mathrm{OC}_{2} \mathrm{H}_{5}\right)_{4}\right)$ forms silanol groups. The condensation or polymerization between the silanol groups or between the silanol groups and the ethoxy groups creates siloxane bridges ( $\mathrm{Si}-\mathrm{O}-\mathrm{Si}$ ), forming the silica structure ${ }^{14}$.

For the second step, two models were developed to describe the mechanism of silica growth: the monomer addition model and the controlled aggregation model. The monomer addition model describes that from the nucleation, growth arises from the addition of hydrolyzed monomers. In turn, the aggregation model assumes that nucleation occurs continuously, and the molecules formed are unified to form dimers, trimers and secondary particles. Both models lead to the formation of spherical or gel silica particles, depending on the reaction conditions ${ }^{14}$.
Parameters such as the concentration of TEOS, alcohol, hydroxides and water, time, temperature, drying stage and mainly the dispersion of the nanoparticles are still under study to guarantee the formation, morphology and size control of silica nanoparticles, as well as the final properties of these materials ${ }^{14,17}$.

The focus of this work was the development of a superhydrophobic self-cleaning surface using perfluorooctyltriethoxysilane (POTS) functionalized silica nanoparticles on a polymeric polymethyl methacrylate (PMMA) substrate using a simple method of preconditioning the substrate with acetone to anchor the nanoparticles. The use of POTs in the functionalized process can propose the electronegative surface caused by eight fluorine in the POTs' chain. The fluorine disposal in the silica surface change the characteristic hydrophilic to hydrophobic.

\section{Experimental}

The experimental section was divided in four parts: the silica nanoparticle synthesis; the deposition of silica in PMMA board; the functionalization of silica and surface modification; and the assessment of the degree of hydrophobicity and self-cleaning of the surface.

\subsection{Silica nanoparticle synthesis}

Silica nanoparticles were prepared by referring to the classic Stöber Method ${ }^{16}$ which involves the suspension stage of tetraethylorthosilicate (TEOS $-\mathrm{Si}\left(\mathrm{OC}_{2} \mathrm{H}_{5}\right)_{4}, 98 \%$ purity) in alcoholic medium (ethyl alcohol, $99.5 \%$ purity). For this purpose, was adopted a mass fraction of $30 \%$ of TEOS and $70 \%$ of anhydrous ethyl alcohol for the controlled hydrolysis of TEOS.

This suspension was dripped into an alkaline matrix containing, in mass fraction values, $18 \%$ of ammonium hydroxide, $37 \%$ of ethyl alcohol and completed with water. This process was considered green chemistry, because it answer some factors like not generate waste; not use toxic and hazardous solvents or reagents and it was not necessary to use higher temperature, like a pirometalurgy process need temperature above $500^{\circ} \mathrm{C}^{20}$. These factors were investigated by Matharu and Lokesh (2019) in life cycle assessment or analysis (LCA) ${ }^{19}$.

In this process, the silica nanoparticle was synthetized and the reactions are represented by equations 1,2 and $3^{21}$ :

- Hydrolysis:

$$
\equiv \mathrm{Si}-\mathrm{OR}+\mathrm{H}_{2} \mathrm{O} \rightleftharpoons \mathrm{Si}-\mathrm{OH}+\mathrm{ROH}
$$

- Condensation in alcohol:

$$
\equiv \mathrm{Si}-\mathrm{OR}+\mathrm{HO}-\mathrm{Si} \equiv \rightleftharpoons \equiv \mathrm{Si}-\mathrm{O}-\mathrm{Si} \equiv+\mathrm{ROH}
$$

- Condensation in water

$$
\equiv \mathrm{Si}-\mathrm{OH}+\mathrm{HO}-\mathrm{Si} \equiv \rightleftharpoons \mathrm{Si}-\mathrm{O}-\mathrm{Si} \equiv+\mathrm{H}_{2} \mathrm{O}
$$


Observing that $\mathrm{R}$ represents an alkyl group $\mathrm{C}_{\mathrm{x}} \mathrm{H}_{2 \mathrm{x}+1}$, as is the case of TEOS $\left(\mathrm{Si}\left(\mathrm{OC}_{2} \mathrm{H}_{5}\right)_{4}\right)$.

Addition of alcohol is necessary since alkoxysilanes are not water soluble. The hydrolysis reaction (1) replaces the alkoxide group (-OR) with hydroxide $(-\mathrm{OH})$ and is catalyzed by ammonium hydroxide. Formation of siloxanes by condensation occurs both in the alcoholic medium and in the aqueous medium.

Generally, the condensation reactions (2) and (3) begin before the hydrolysis reaction (1) comes to an end.

This matrix solution was kept under stirring while adding the alcohol solution of TEOS (previously prepared).

This mixture remains under constant stirring for 2 hours at $(25 \pm 2)^{\circ} \mathrm{C}$. At the end of the 2 -hour stirring, the hydrolyzed silica is obtained (in the range of $400 \mathrm{~nm}$ to $800 \mathrm{~nm}$ ).

The hydrolyzed silica (silica nanoparticles) was separated from the rest of the solution by centrifugation for 30 minutes at 7,000 rpm. The supernatant was discarded, and the silica washed with distilled water and centrifuged again for 20 minutes. This washing procedure was repeated two more times to remove residual hydroxide and alcohol. The solid obtained from silica was oven dried at $(150 \pm 2){ }^{\circ} \mathrm{C}$ for 24 hours. The lower temperature favors the smaller particle while higher temperature favors the bigger one ${ }^{22}$.

\subsection{Silica nanoparticle deposition}

Latthe (2018) observed poor adhesion of silica nanoparticles on glass ${ }^{23}$, so roughness was created directly on the boards for better adhesion of the layers to be created using acetone (acetone, 100\% purity). Xing et al (2017) used nitric acid to treat polycarbonate to create a surface roughness due to the formation of $\mathrm{C}=\mathrm{O}$, Si-O-C, and $\mathrm{Si}-\mathrm{O}-\mathrm{Si}$ groups, increasing the superhydrophobicity ${ }^{24}$.

For the nanoparticles deposition, a $2 \mathrm{~cm} \mathrm{\times} 6 \mathrm{~cm}$ polymethylmethacrylate (PMMA) board was used, in which the aqueous suspension containing $1 \%$ by weight of silica nanoparticles suspended in acetone (acetone, $100 \%$ purity) was dripped.

As a step of preparation of the aqueous suspension of silica nanoparticles, ultrasonic equipment was used for 1 hour for rupturing silica agglomerates, facilitating the deposition of silica nanoparticles in the substrate (PMMA), forming a monophasic nanocomposite.

The preparation of this substrate was divided into two parts: the deposition of the silica nanoparticle solution and the deposition of the solution of $1 \mathrm{H}, 1 \mathrm{H}, 2 \mathrm{H}, 2 \mathrm{H}-$ perfluorooctylethoxysilane (POTS). The acetone solution containing $1 \%$ silica nanoparticles was first deposited with the aid of a Pasteur pipette over the entire substrate surface ${ }^{25}$.

Three applications were performed and, for each application, the solution dried naturally on the PMMA surface. The conditions was $25^{\circ} \mathrm{C}+/-2^{\circ} \mathrm{C}$ with range of 24 $\mathrm{h}$ to another application.

\subsection{Silica nanoparticle functionalization and surface modification}

Although the silica nanoparticles have hydroxyls in their structure and is characterized as hydrophilic, these particles can be applied in the formation of hydrophobic surfaces from their functionalization, made with the creation of another layer with the objective of reducing free surface energy ${ }^{26}$.

The silica nanoparticles functionalization was made to increase the hydrophobicity by the presence of functional groups with fluorine, which have electronegative character. The functionalization is known to have two processes: cocondensation method and post-grafting method ${ }^{17,27}$.

The co-condensation method deals with the simultaneous synthesis and functionalization of the silica nanoparticles. In other words, in a single step, the hydrolysis and condensation take place together with the silica precursor and the organic compound.

In the functionalization by post-grafting method, the organic molecule in the nanoparticle is initially immobilized and then synthesized with an organic agent. For this, a solution of $0.5 \mathrm{wt} . \%$ of POTS in ethanol ( $99.5 \%$ purity) was used. The POTS solution was heated to $65^{\circ} \mathrm{C} \pm 5^{\circ} \mathrm{C}$ for 30 minutes under agitation ${ }^{28}$.

All glassware and instruments used in this phase are made from polytetrafluoroethylene (PTFE) or polypropylene (PP), in order to avoid the early deposition of silica nanoparticles.

The silica nanoparticles preparation and deposition are shown in Figure 1.

In this stage of the process, the surface of the PMMA substrate has a hydrophilic character, due to the hydrogen bonds on the surface of the silica nanoparticles layer deposited and, therefore, they present a polar character.

After drying, the solution of POTS can be applied by dripping, whereby the functionalization of the silica nanoparticles occurs. This functionalization is caused by the nucleophilic attack of the hydroxyl group (from the $\mathrm{SiO}_{2}$ surface) to the silicon atom ( $\mathrm{Si}$ ) of the POTS molecule, as shown in Figure 2.

From this reaction, an ethanol molecule was released and evaporates during the drying of the film. Therefore, after functionalization, the surface acquires a hydrophobic character.

\subsection{Characterization}

After the functionalization of the surface materials, the degree of hydrophobicity of the material was evaluated using a CA Model 100-00 goniometer (Ramé-Hart Inc., USA) to take measure of the contact angle between a sessile drop and the surface using the Young-Laplace method, the surface energy and the self-cleaning capacity of the modified layer. The static and dynamic contact angle were measured, with the table horizontally and tilting the table, respectively. The tests were performed with deionized water. 

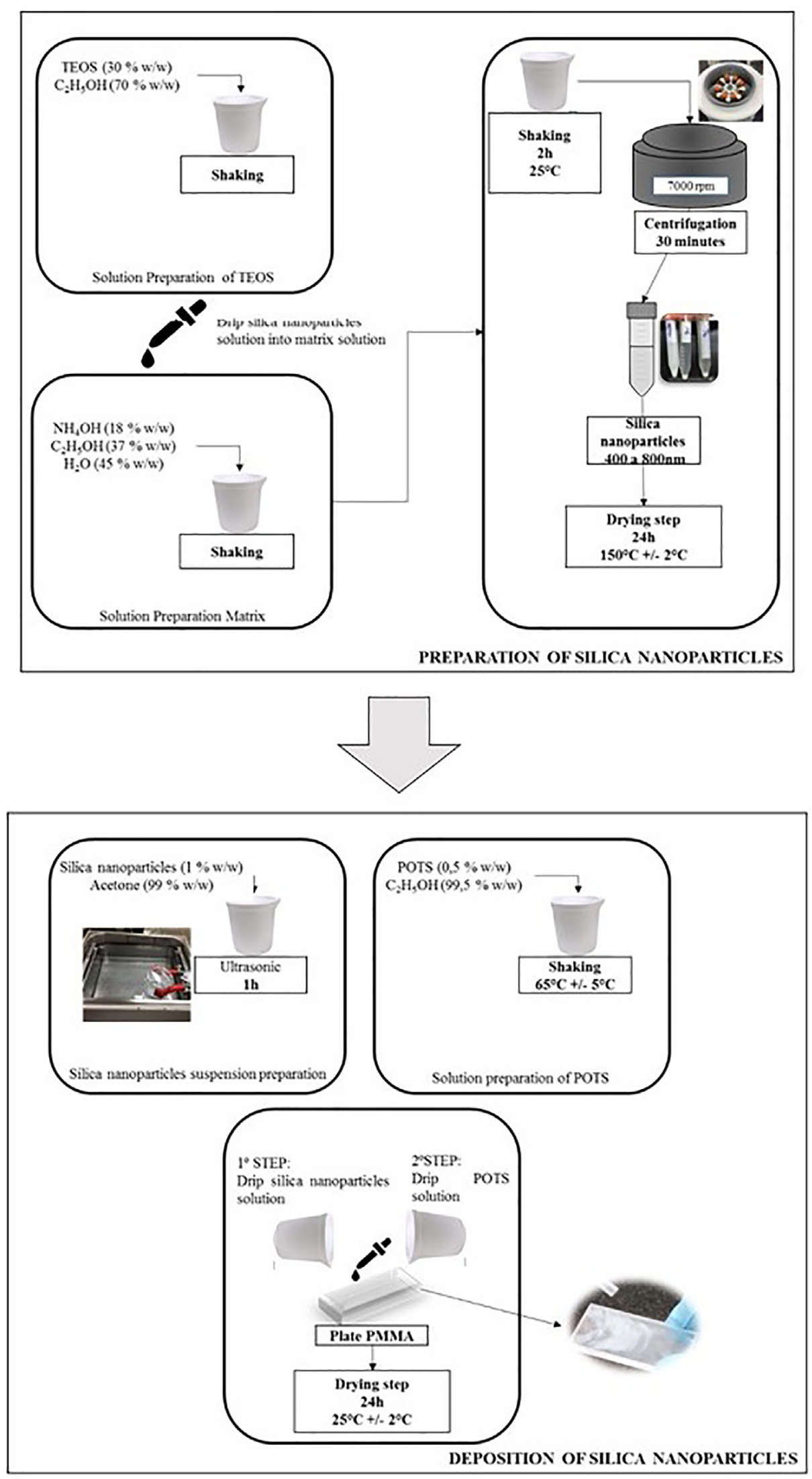

Figure 1. Silica nanoparticle preparation and deposition process flowchart. 

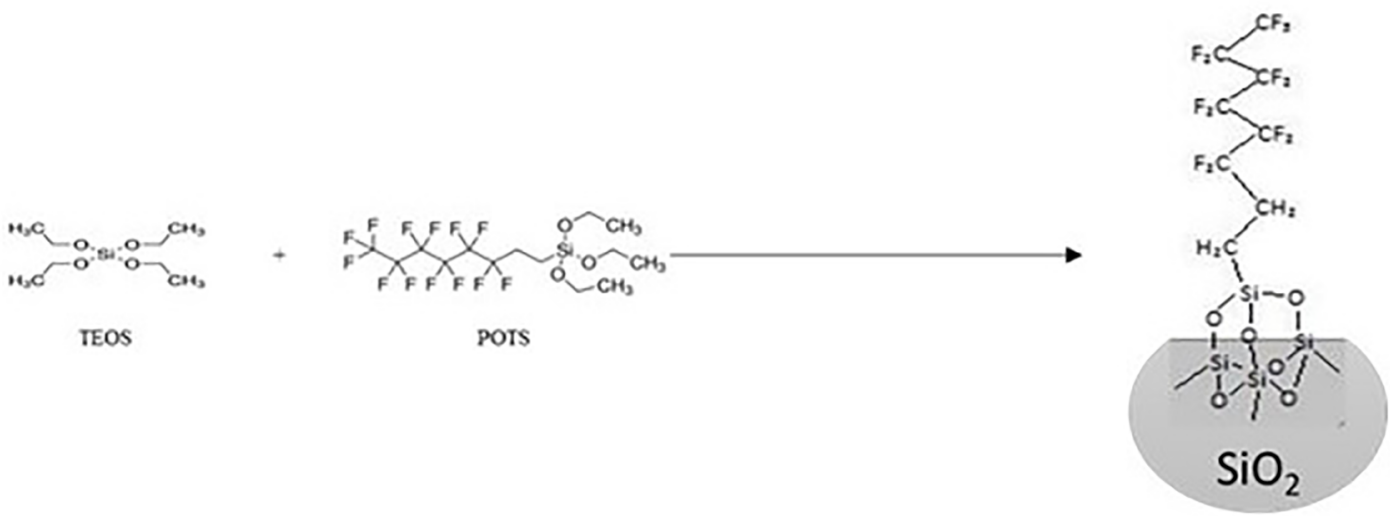

Figure 2. Representative scheme of polycondensation reaction.

This procedure was repeated ten times in several regions of the board, randomly selected to obtain a mean value. Hence, the homogeneity of the deposit on the surface of the PMMA plate can be evaluated.

PMMA substrates with different surface modified treatments were analyzed:

- untreated PMMA;

- $\quad$ PMMA + silica nanoparticles (NP);

- PMMA + POTS and

- $\quad \mathrm{PMMA}+$ silica nanoparticles (NP) + POTS.

For measuring the surface energy, in the experimental procedure with the goniometer, diiodomethane $\left(\mathrm{CH}_{2} \mathrm{I}_{2}\right)$ was used. The tests were carried out in a controlled environment with room temperature at $(20 \pm 2){ }^{\circ} \mathrm{C}$.

The self-cleaning capacity was evaluated by spreading $10 \mathrm{mg}$ of powder charcoal on the modified surface and dropping a $10 \mu 1$ water drop on the surface using the goniometer. Water droplets form a round shape on the superhydrophobic surface and flow quickly simply by contacting the surface. Water adhesion is more intense with dust than dust adhesion with the superhydrophobic surface. Thus, spherical drops of water collect dust particles abundantly and clean the surface ${ }^{29,30,31,32}$. A similar test was performed by tilting the board with powder charcoal and observing the falling drops.

The mechanical stability of the modified surface was qualitatively analyzed by water jet impact. A $25 \mathrm{ml}$ capacity syringe was completely filled with methylene blue colored water. Manually the syringe was emptied $3 \mathrm{~cm}$ above the sample surface, impacting the surface. An EOS T5i Canon digital camera was used to capture optical images of the surface.

The surface materials characterization was conducted by scanning electron microscopy -SEM (ProX Desktop SEM, Phenom-World BV) to verify the formation of silica nanoparticles (NP) and the modified surface.

\section{Results and Discussion}

The results were presented after the morphological characterization of the silica nanoparticles synthesized via sol-gel method: functionalization and deposition of silica nanoparticles; and evaluation of the degree of hydrophobicity, surface energy and self-cleaning.

\subsection{Morphological characterization of synthesized silica nanoparticles}

Silica nanoparticles (NP) synthesis by the sol-gel process from TEOS showed a mean value of particle size in the range of $400 \mathrm{~nm}$ to $800 \mathrm{~nm}$, as seen in Figure 3.

In the silica-nanoparticle synthesis via sol-gel method, the predominance in the formation of particles with a diameter between $400 \mathrm{~nm}$ and $500 \mathrm{~nm}$ was observed, which can favor the performance in terms of hydrophobicity, because the smaller the average particle size, the greater the area of contact and the greater reaction site. The reaction site favors subsequent functionalization that in turn will provide greater hydrophobicity ${ }^{17}$.

\subsection{Morphological characterization of silica nanoparticle deposition}

The surface materials formed by the deposition of functionalized silica nanoparticles and POTS in the substrate of PMMA is shown in Figure 4. The modified surface showed a homogeneous deposition.

According to the Cassie-Baxter model, super-hydrophobicity was achieved only with the optimization of surface topography, where microstructures uniformly dispersed in the surface space create air pockets below the deposited droplets, decreasing their direct contact with the surface ${ }^{33}$. Among the various substances available, silica is the most used for the manufacture of nanoparticles, due to the simplicity of the synthesis method, biocompatibility, uniform particle diameter, practical surface particle distribution, among other factors ${ }^{34,35}$.

\subsection{Assessment of the degree of hydrophobicity and self-cleaning}

The results of the static water contact angle and the surface energy of the samples were presented in Table 1. 


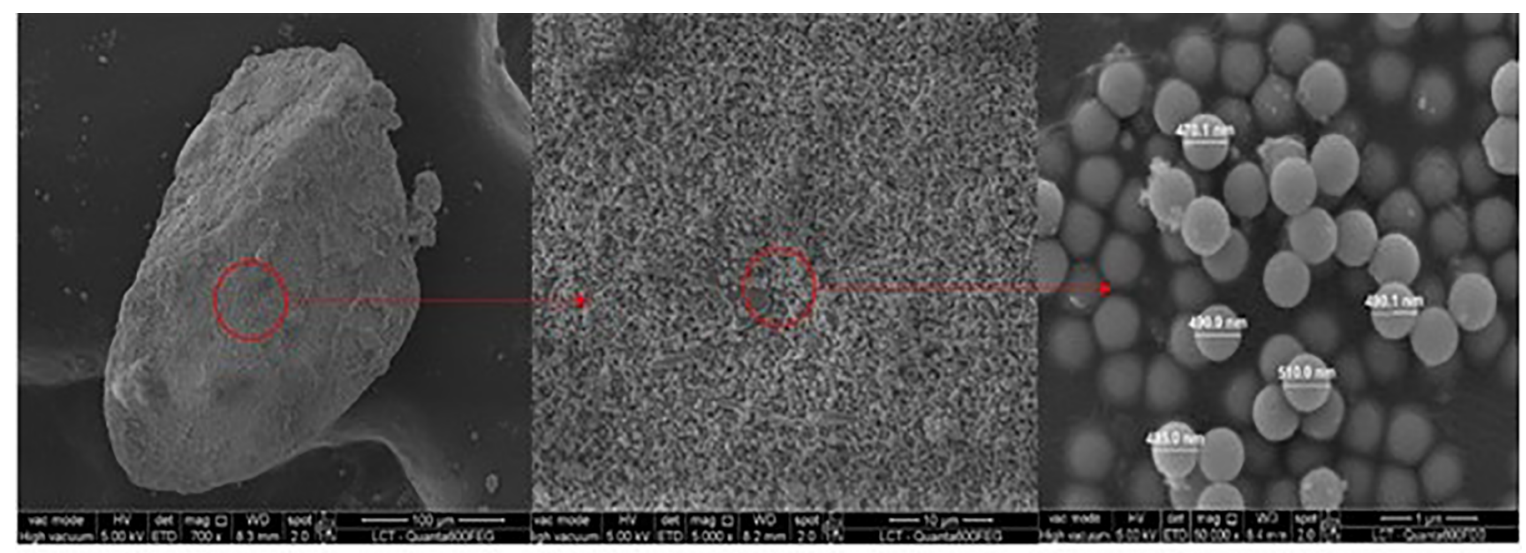

(a)

(b)

(c)

Figure 3. Micrography of the silica nanoparticles synthesized via sol-gel from TEOS, performed in a Scanning Electron Microscope (SEM). (a) magnification by 200 times; (b) magnification by 5,000 times; (c) magnification by 50,000 times.

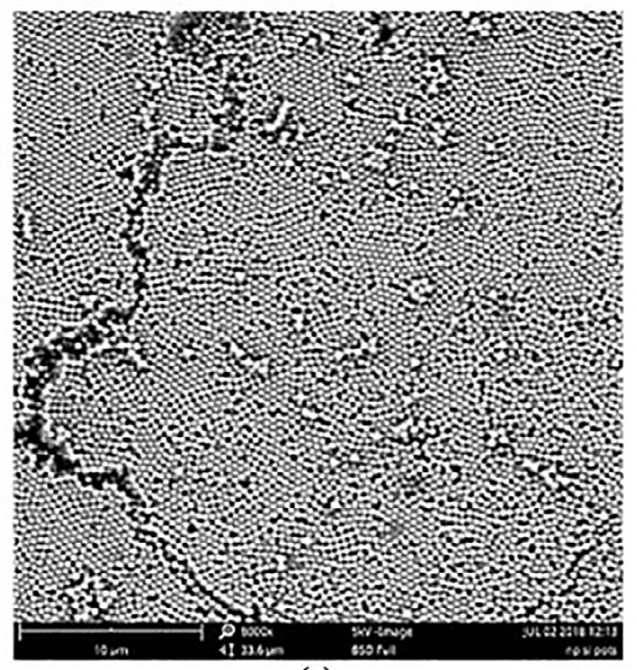

(a)

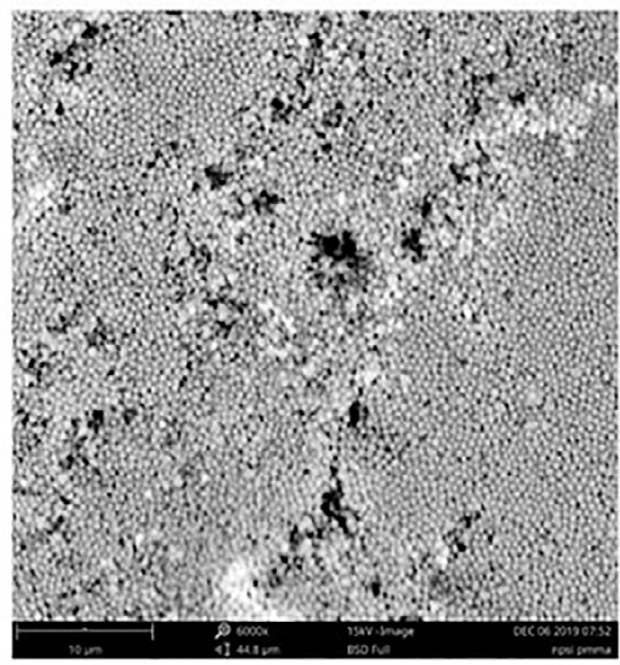

(c)

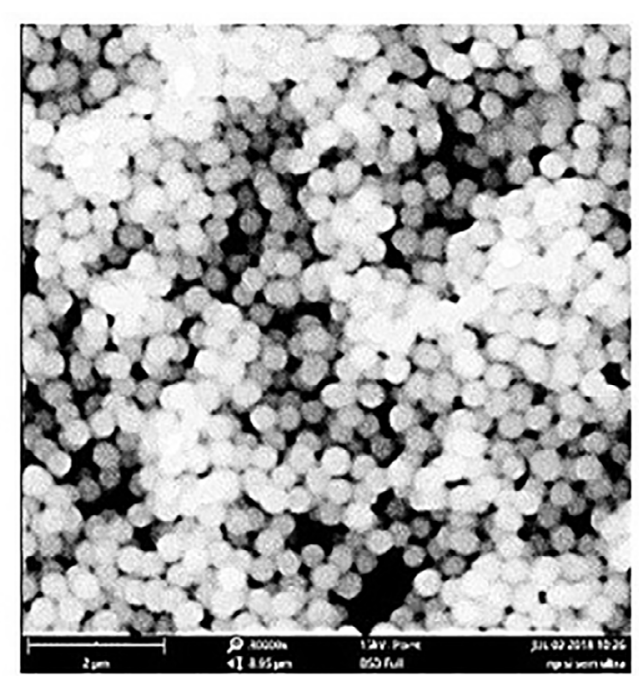

(b)

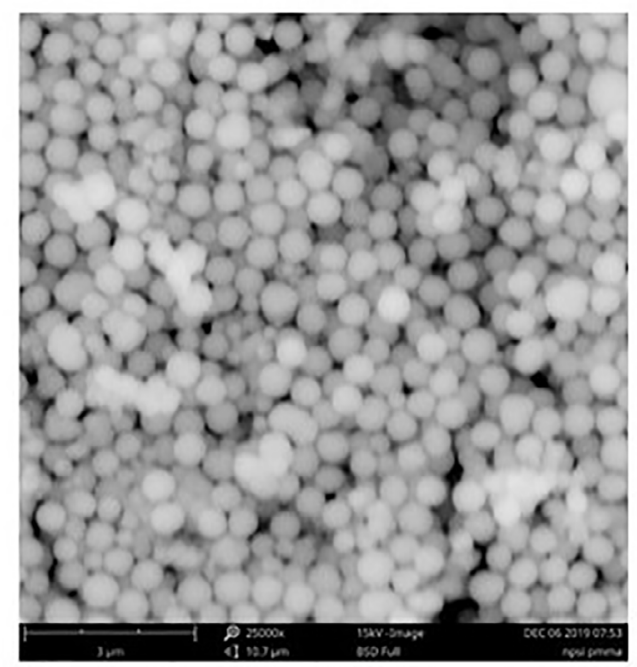

(d)

Figure 4. Micrography of the silica nanoparticles on a PMMA board, performed in a SEM. (a) functionalized with POTS magnification by 5,500 times; (b) functionalized with POTS magnification by 30,000 times; (c) not functionalized magnification by 6,000 times; (d) not functionalization magnification by 25,000 times. 
Table 1. Average apparent contact angle and surface energy of PMMA substrates subjected to surface treatment.

\begin{tabular}{lcc}
\hline Samples & $\begin{array}{c}\text { Static contact } \\
\text { angle }\left({ }^{\circ}\right)\end{array}$ & $\begin{array}{c}\text { Surface energy } \\
(\mathbf{d y n} / \mathbf{c m})\end{array}$ \\
\hline Untreated PMMA & $77.2 \pm 1.1$ & $33.00 \pm 0.02$ \\
PMMA + NP & n.d. & n.d. \\
PMMA + POTS & $115.7 \pm 1.6$ & $12.79 \pm 0.77$ \\
PMMA + NP + POTS & $150.32 \pm 0.44$ & $1.45 \pm 0.02$ \\
\hline
\end{tabular}

n.d. $=$ not determined

The PMMA + NP sample has no contact angle results and consequently no surface energy, since it's very hydrophilic. Similar results were obtained by $\mathrm{Yu}(2019){ }^{17}$ with an unmodified silica nanoparticle surface compared with a functionalized silica nanoparticle surface. The contact angle result of the unmodified surface was only $20^{\circ}$, because the large number of hydroxyl groups made the surface hydrophilic, while the contact angle of the modified surface was $136^{\circ}$.

The dynamic contact angle for the PMMA + NP + POTS sample performed with water was $(7.5 \pm 0.38)$ degrees, that is, the drop rolls abruptly as the goniometer table was tilted at this angle, characterizing the surface as superhydrophobic ${ }^{17,25,26,27}$. The other surfaces did not show a dynamic angle; even when the board was at a 90 degrees angle, the water drops did not roll.

The treatment performed with silica nanoparticles and POTS (sample PMMA + NP + POTS with surface energy equal to $1.45 \pm 0.02 \mathrm{dyn} / \mathrm{cm}$ ) reduces the surface energy of untreated PMMA (surface energy equal to $33.00 \pm 0.02 \mathrm{dyn} /$ $\mathrm{cm}$ ) by $95.6 \%$, making the treatment much more efficient and hydrophobic with self-cleaning properties.

The surface free energy (SEF) was calculated by the software Advance - DropShape module and 9 models were considered for this calculation, for example, the geometric mean, the harmonic mean, the Lifshitz-van der Waals/acidbase models. This software considers the morphology and contact angle using water and diiodomethane ${ }^{36}$.

The lower the free energy of the surface, the greater the hydrophobicity of the material. It can be observed when comparing the different treatments given to the PMMA board. The untreated PMMA has shown a contact angle lower than 90 degrees and surface energy equal to $33 \mathrm{dyn} /$

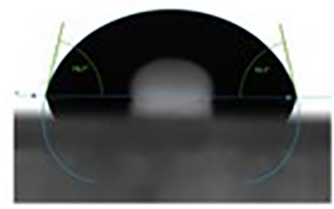

Untreated PMMA

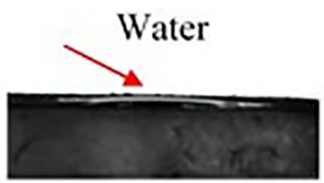

PMMA+NP (a) (b) $\mathrm{cm}$, characterizing a hydrophilic behavior. When it comes to the PMMA with POTS deposited, its behavior changes to hydrophobic and when it's deposited silica nanoparticles besides POTS on the board, its behavior changes to superhydrophobic, resulting in a 150 degrees contact angle and surface energy of $1.45 \mathrm{dyn} / \mathrm{cm}^{32}$.

Figure 5 shows the droplet on the substrates with images obtained with the goniometer camera, illustrating the wettability of the surface. The figure also allows verifying how spherical the droplets are on the surface of the PMMA board; PMMA board treated with POTS and PMMA board with silica nanoparticles and POTS, which indicates the hydrophobic character of the surface.

The PMMA substrate treated with the functionalized silica nanoparticles showed apparent static contact angle values around 151 degrees, this is, higher than 90 degrees, since the coupling agent molecules (POTS) present functional groups containing fluorine $(\mathrm{F})$ in the carbonic chain. Fluorine is electronegative, creating an electronic cloud at bond with carbon (dipole), which causes the effect of repelling polar substances like water and, therefore, hydrophobicity on the surface of the PMMA substrate ${ }^{37}$. By increasing the mass ratio of functionalized silica nanoparticles in an acrylic nanocomposite from 1 to 2, $\mathrm{Li}$ (2019) found an increase in the contact angle of the composite coating from 103 to 152 degrees ${ }^{38}$

The self-cleaning property of the superhydrophobic modified surface with PMMA as substrate and deposition of NP and POTS has been verified. An amount of $10 \mathrm{mg}$ of coal powder was spread on the surface as shown in Figure 6a. A $10 \mu \mathrm{l}$ drop of water was produced from the goniometer needle and slowly deposited over the dusty area. When the goniometer table was tilted, the water droplets rolled rapidly, dusting the surface and erupting after leaving the surface (Figure 6b). The same effect can be observed when the table was kept horizontal (Figure 6c). A drop of water can easily take out all the carbon particles along their way, confirming the self-cleaning ability of the superhydrophobic surface ${ }^{27}$.

The mechanical durability of the surface was qualitatively evaluated by the water jet test. The water jet was immediately repelled from the surface (Figure $7 \mathrm{a}$ ) and the constant water local tapping did not damage the surface as well as its superhydrophobicity (Figure 7b).

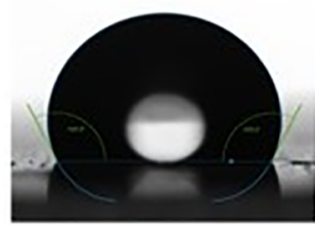

PMMA+POTS

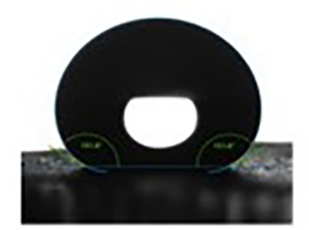

$\mathrm{PMMA}+\mathrm{NP}+\mathrm{POTS}$ (c)

Figure 5. Image of the water test performed on the PMMA substrates after the deposition of nanoparticles, obtained with the goniometer 100-00 camera to calculate the apparent static contact angle. 


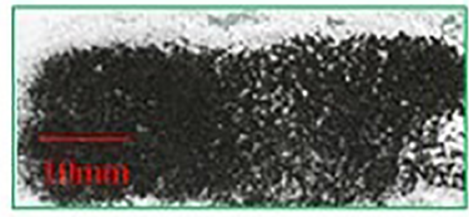

(a)

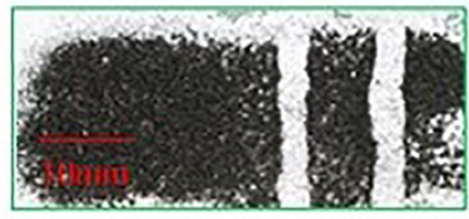

(b)

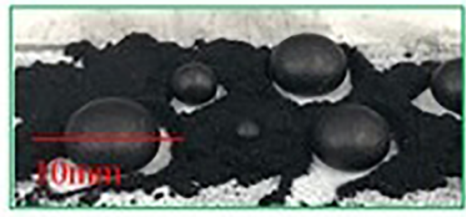

(c)

Figure 6. Self-cleaning performance of the modified surface (PMMA+NP+POTS). (a) coal powder on the surface, (b) self-cleaning performance on tilted surface, (c) self-cleaning performance on horizontal position.

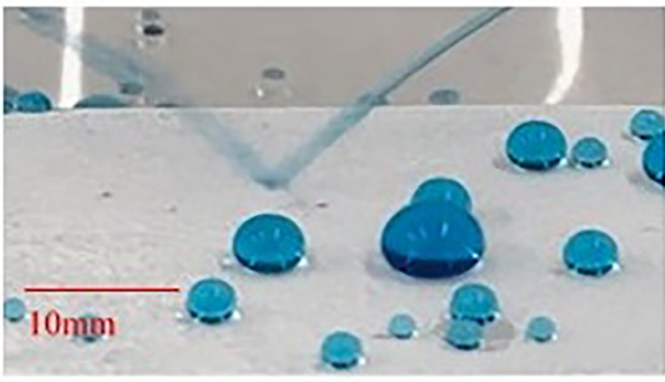

(a)

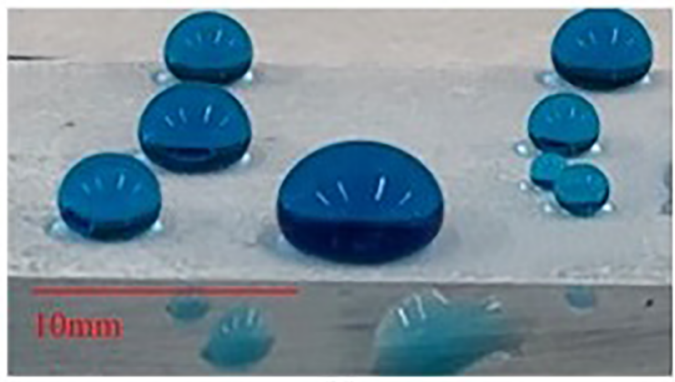

(b)

Figure 7. Optical photograph of (a) water jet bouncing on the superhydrophobic modified substrate, (b) water drops on the superhydrophobic modified substrate after water jet bouncing.

Water jets hit the surface repeatedly when 5 full syringes were spilled onto the surface, however, the contact angle values remained unchanged, as well as the spheroidicity of the water droplets.

Liu et al (2015) found to a water drops spherical shape on this surface glass with a contact angle of $169^{\circ}$. The process used to fabricate self-cleaning superhydrophobic coatings was the sol-gel process using fluoroalkylsilane ${ }^{39}$. Pawar et al (2017) used silica particles, methyltrichorosilane and tetrahydrofuran in a polystyrene surface to get a contact angle of $158^{\circ}$, producing a superhydrophobicity coating ${ }^{40}$.

\section{Conclusion}

According to the tests performed and the results from the functionalization of silica nanoparticles on the PMMA board, the technique was observed to be effective in obtaining a hydrophobic and self-cleaning surface, as it obtained an average static contact angle of $(150.32 \pm 0.44)$ degrees, and a dynamic contact angle smaller than 8 degrees.

The functionalization with POTS of the silica nanoparticles on the surface of the PMMA board was shown to be essential for obtaining hydrophobic surfaces since before applying the functionalization treatment, the surface of the nanoparticles was hydrophilic due to the hydrogen bonds. Thus, after the treatment, due to carbon-fluorine bonds, the contact angle showed an increase of $52.75^{\circ}$

In relation to surface energy, a low value was obtained, as expected, of $(1.45 \pm 0.02) \mathrm{dyn} / \mathrm{cm}$, representing a decrease by $95.6 \%$ as compared to a PMMA-only board (without treatment).
The modified superhydrophobic surface revealed a superior self-cleaning performance by freely rolling spherical water drops on the non-wettable surface. The stability of the modified surface has been proven by water jet impact.

\section{Acknowledgment}

Mauá Institute of Technology - IMT (São Caetano do Sul - SP, Brazil)

LAREX - Laboratory of Recycling, Waste Treatment and Extraction to the Department of Chemical Engineering of the Escola Politécnica of the University of São Paulo (POLI-USP)

Technological Plasma Laboratory of UNESP in Sorocaba (SP, Brazil)

\section{References}

1. Malshe A, Rajurkar K, Samant H, Hansen HN, Bapat S, Jiang W. Bio-inspired functional surfaces for advanced applications. CIRP Annals - Manufacturing Technology. 2013;62(2):607-628.

2. Feng L, Li S, Li Y, Li H, Zhang L, Zhai J, et al. Super-hydrophobic surfaces: from natural to artificial. Advanced Materials. 2002;14(24):1857-1960

3. Wenzel RN. Resistance of solid surfaces to wetting by water. Industrial and Engineering Chemistry Research. 1936;28(8):988-994.

4. Li X, Du X, He J. Self-cleaning antireflective coatings assembled from peculiar mesoporous silica nanoparticles. Langmuir. 2010;26(16):13528-13534. 
5. Ahuja A, Taylor JA, Lifton V, Sidorenko AA, Salamon TR, Lobaton EJ, et al. Nanonails: a simple geometrical approach to electrically tunable superlyophobic surfaces. Langmuir. 2008 Jan;24(1):9-14.

6. Tian H, Wang F, Ge S, Ou J, Li W, Yu S. A simple and effective way to fabricate mechanical robust superhydrophobic surfaces. RSC Advances. 2016;6(34):28563-28569.

7. Li H, Yu S. A robust superhydrophobic surface and origins of its self-cleaning properties. Applied Surface Science. 2017;420:336-345.

8. Tesler AB, Kim P, Kolle S, Howell C, Ahanotu O, Aizenberg J. Extremely durable biofouling-resistant metallic surfaces based on electrodeposited nanoporous tungstite films on steel. Nature Communications. 2015;6:8649.

9. Qiang S, Chen K, Yin Y, Wang C. Robust UV-cured superhydrophobic cotton fabric surfaces with self-healing ability. Materials and Design. 2017;116:395-402.

10. Li D, Guo Z. Stable and self-healing superhydrophobic $\mathrm{MnO} 2$ fabrics: applications in self- cleaning, oil/water separation and wear resistance. Journal of Colloid and Interface Science. 2017;503:124-130.

11. Zhang L, Xue CH, Cao M, Zhang MM, Li M, Ma JZ. Highly transparent fluorine-free superhydrophobic silica nanotube coatings. Chemical Engineering Journal. 2017;320:244-252.

12. Drelich J, Marmur A. Physics and applications of superhydrophobic and superhydrophilic surfaces and coatings. Surface Innovations. 2014;2(4):211-227.

13. Latthe SS, Sutar RS, Bhosale AK, Sadasivuni KK, Liu S. Superhydrophobic surfaces for oil-water separation. Superhydrophobic Polymer Coatings. 2019;339-356.

14. Latthe SS, Sutar RS, Shinde TB, Pawar SB, Khot TM, Bhosale AK, et al. Superhydrophobic leaf mesh decorated with $\mathrm{SiO}_{2}$ nanoparticle-polystyrene nanocomposite for oil-water separation. ACS Applied Nano Materials. 2019;2(2):799-805.

15. Rahman IA, Padavettan V. Synthesis of silica nanoparticles by sol-gel: size-dependent properties, surface modification, and applications in silica-polymer nanocomposites - a review. Journal of Nanomaterials. 2012;2012:1687-41110.

16. Zou H, Wu S, Shen J. Polymer/silica nanocomposites: preparation, characterization, properties, and applications. Chemical Reviews. 2008;108(9):3893-957.

17. Inagaki S, Guan S, Fukushima Y, Ohsuna T, Terasaki O. Novel mesoporous materials with a uniform distribution of organic groups and inorganic oxide in their frameworks. Journal of the American Chemical Society. 1999;121(41):9611-9614.

18. Linderverg R, Sjöblom J, Sundholm G. Preparation of silica particles utilizing the sol-gel and the emulsion-gel processes. Colloids and Surfaces A: Physicochemical and Engineering Aspects. 1995;99(1):79-88.

19. Stober W, Fink A, Bohn E. Controlled growth of monodisperse silica spheres in the micron size range. Journal of Colloid and Interface Science. 1967;26:62-69.

20. Matharu AS, Lokesh K. Green chemistry principles and global drivers for sustainability: an introduction. In: Höfer R, Matharu AS, Zhang Z. Green Chemistry for Surface Coatings, Inks and
Adhesives: Sustainable Applications. London, UK: Royal Society of Chemistry; 2019. p. 1-17.

21. Brinker CJ, Scherer GW. Sol-gel science: the physics and chemistry of sol-gel processing. New York: Academic Press; 1990.

22. Almkhelfe H, Amama PB. Supercritical fluids as reaction media for scalable production of carbon nanomaterials. ACS Applied Nano Materials. 2019;2(2):1009-1017.

23. Latthe SS, Sutar RS, Kodag VS, Bhosale AK, Kumar AM, Sadasivuni KK, et al. Self - cleaning superhydrophobic coatings: potential industrial applications. Progress in Organic Coatings. 2019;128:52-58.

24. Xing R, Latthe SS, Bhosale AK, Li R, Kumar AM, Liu S. A novel and facile approach to prepare self-cleaning yellow superhydrophobic polycarbonates. Journal of Molecular Liquid. 2017;247:366-373.

25. Wang S, Liu C, Liu G, Zhang M, Li J, Wang C. Fabrication of superhydrophobic wood surface by a sol-gel process. Applied Surface Science. 2011;258(2):806-810.

26. Yu K, Liang Y, Ma G, Yang L, Wang TJ. Coupling of synthesis and modification to produce hydrophobic or functionalized nano-silica particles. Colloids and Surfaces A: Physicochemical and Engineering Aspects. 2019;574:122-130.

27. Melde BJ, Holland BT, Blanford CF, Stein A. Mesoporous sieves with unified hybrid inorganic/organic frameworks. Chemistry of Materials. 1999;11(11):3302-3308.

28. Kang S, Hong S, Choe CR, Park M, Rim S, Kim J. Preparation and characterization of epoxy composites filled with functionalized nanossílica particles obtained via sol-gel process. Polymer Science. 2001;42:879- 887.

29. Liu S, Liu X, Latthe SS, Gao L, An S, Yoon SS, et al. Selfcleaning transparent superhydrophobic coatings through simple sol-gel processing of fluoroalkylsilane. Applied Surface Science. 2015;351:897-903.

30. Xing R, Latthe SS, Bhosale AK, Li R, Kumar AM, Liu S. A novel and facile approach to prepare self-cleaning yellow superhydrophobic polycarbonates. Journal of Molecular Liquids. 2017;247:366-373.

31. Latthe SS, Sutar RS, Kodag VS, Bhosale AK, Kumar AM, Sadasivuni KK, et al. Self- cleaning superhydrophobic coatings: potential industrial applications. Progress in Organic Coatings. 2019;128:52-58.

32. Kokare AM, Sutar RS, Deshmukh SG, Xing R, Liu S, Latthe SS. ODS - modified TiO2 nanoparticles for the preparation of self-cleaning superhydrophobic coating. In: $2^{\text {nd }}$ International Conference on Condensed Matter and Applied Physics (ICC 2017); 2017 nov 24- 25; Bikaner, India. Bikaner (IND): AIP Conference Proceedings; 1953. p. 100068.

33. Cassie ABD, Baxter $\mathrm{S}$. Wettability of porous surfaces. Transactions of the Faraday Society. 1944;40:546-551.

34. Moon S, Lee KJ. Simultaneous control of size and surface functionality of silica particle via growing method. Advanced Powder Technology. 2017;28(11):2914-920.

35. Cengiz U, Cansoy CE. Applicability of Cassie-Baxter equation for superhydrophobic fluoropolymer-silica composite films. Applied Surface Science. 2015;335:99-106. 
36. Namen F, Galan Junior J, Oliveira JF, Cabreira RD, Silva Filho FC, Souza AB, et al. Surface properties of dental polymers: measurements of contact angles, roughness and fluoride release. Materials Research. 2008;11(3):239-243.

37. Schuster JM, Schvezov CE, Rosenberger MR. Analysis of the results of surface free energy measurement of Ti6Al4V by different methods. Procedia Materials Science. 2015;8:732-741.

38. Li M, LiY, XueF, Jing X.A robust and versatile superhydrophobic coating: wear resistance study upon sandpaper abrasion. Applied Surface Science. 2019;480:738-48.
39. Liu S, Liu X, Latthe SS, Gao L, An S, Yoon SS, Liu B, Xing R. Self-cleaning transparent superhydrophobic coatings through simple sol-gel processing of fluoroalkylsilane. Applied Surface Science. 2015;351:897-903.

40. Pawar PG, Xing R, Kambale RC, Kumar AM, Liu S, Latthe SS. Polystyrene assisted superhydrophobic silica coatings with surface protection and self-cleaning approach. Progress in Organic Coatings. 2017;105:235-244. 